\title{
Using the Model of Resource and Time-Based Triage (MORTT) to Guide Scarce Resource Allocation in the Aftermath of a Nuclear Detonation
}

Rocco Casagrande, PhD; Nick Wills, MS; Elizabeth Kramer, BS; Louise Sumner, BS;

Mark Mussante, BS; Rachel Kurinsky, BS; Patrick McGhee, BS; Luba Katz, PhD;

David M. Weinstock, MD; C. Norman Coleman, MD

\section{ABSTRACT}

Conventional triage algorithms assume unlimited medical resource availability. After a nuclear detonation, medical resources are likely to be particularly limited, suggesting that conventional triage algorithms need to be rethought. To test various hypotheses related to the prioritization of victims in this setting, we developed the model of resource- and time-based triage (MORTT). This model uses information on time to death, probability of survival if treated and if untreated, and time to treat various types of traumatic injuries in an agent-based model in which the time of medical practitioners or materials can be limited. In this embodiment, MORTT focuses solely on triage for surgical procedures in the first 48 hours after a nuclear detonation. MORTT determines the impact on survival based on user-selected prioritization of victims by severity or type of injury. Using MORTT, we found that in poorly resourced settings, prioritizing victims with moderate life-threatening injuries over victims with severe life-threatening injuries saves more lives and reduces demand for intensive care, which is likely to outstrip local and national capacity. Furthermore, more lives would be saved if victims with combined injury (ie, trauma plus radiation $>2$ Gy) are prioritized after nonirradiated victims with similar trauma.

(Disaster Med Public Health Preparedness. 2011;5:S98-S110)

Key Words: triage, modeling, nuclear detonation, improvised nuclear device, combined injury

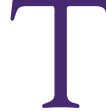
riage is an assessment and sorting process used to prioritize casualties and is historically based upon the medical needs and likelihood of survival of the victims. ${ }^{1}$ Hospital emergency department staff engage in triage processes routinely, determining the treatment order by separating patients requiring immediate medical care from those with less-pressing emergencies. ${ }^{2}$ In a mass casualty incident-whether a multiple vehicle crash, a major hurricane, tornado, or a nuclear detonation - the sheer number of casualties can overwhelm the available medical personnel and health care resources. The focus of triage in a mass casualty incident changes from the needs of an individual victim to the goal of saving the most lives possible. ${ }^{3}$ A mass casualty incident involving a nuclear explosion has the potential to produce catastrophic structural damage and injuries. As described in other articles in this special issue of Disaster Medicine and Public Health Preparedness ${ }^{4-8}$ people with serious trauma combined with burns or radiation injury will require complex care and will have a worse prognosis than people with injuries caused by either trauma or radiation alone. The medical needs

\section{Supplemental digital content available online}

Supplemental digital content is available for this article at http://www.dmphp.org. of these victims will far outweigh the capacities of the available rescue personnel and surrounding medical facilities. Thus, efficient and effective triage will be essential.

\section{AVAILABLE TRIAGE SCHEMES}

Field-based triage systems evaluate individuals on the basis of certain physiological or anatomic criteria and then categorize them into clearly defined groups: those who require immediate lifesaving intervention, those with serious injuries whose condition will not be adversely affected by a delay in short-term treatment, people who are mildly injured, and deceased people. Some triage systems include an additional expectant category for gravely injured individuals who are highly unlikely to survive even with rigorous clinical intervention. In addition, color-coding schemes are frequently used so that the victims in each triage category can be easily discerned by other personnel.

The Simple Triage and Rapid Treatment (START) system is the most widely adopted field triage system for mass casualties used in North America. ${ }^{9}$ A pediatric version of the START protocol (JumpSTART) has been developed for use in children who are 1 to 8 years old, which addresses differences in respiratory physiology be- 
tween adults and children. ${ }^{10}$ START and other triage systems have been used in a number of mass casualty incidents. After the collapse of the World Trade Center towers on September 11, 2001, New York City emergency medical services used the START protocol to triage patients at the scene. ${ }^{11}$ An alternate protocol, Triage Sieve, was used to triage the casualties of the 2005 London Underground bombing, ${ }^{12}$ whereas emergency medical services sorted the casualties of the 2002 Bali nightclub bombings using CareFlite. ${ }^{13}$

Despite their extensive use, the application of these systems to a mass casualty incident is not necessarily backed by an evidencebased rationale. The triage protocols are based on physiological criteria that loosely indicate clinical instability. These systems are used primarily to determine which victims need to be transported to hospitals first and strives to do the most good for the largest number of casualties, but it is difficult to assess success in this respect because the algorithms offer no predictions of expected survivorship. ${ }^{14-16}$ All of these systems consider that enough medical resources are available to provide lifesaving care to those who will benefit from it, regardless of how many victims require it.

In the aftermath of a nuclear detonation, victims will vastly outnumber available medical personnel and require more material resources than are available. Therefore, many of those triaged to a "delayed" category would deteriorate into the "immediate" category well before victims in the original "immediate" category were treated. Furthermore, these systems do not consider that some life-threatening injuries require vastly more time and resources to address than other life-threatening injuries. In addition, none of these systems consider that many badly injured victims (who are still breathing) have a relatively low probability of survival based on the extent of their injuries, even with immediate treatment because the "expectant" category is reserved for those with almost no chance of survival.

The Sacco triage method (STM), a mathematical model that prioritizes casualties in real-time based on the availability of limited medical resources, overcomes some of these concerns. STM is intended to maximize the expected number of survivors based upon the survival probabilities of the casualties. At the scene, emergency personnel evaluate casualties on the basis of respiratory rate, pulse rate, and motor response to generate a respiratory-pulse-motor score. Once victim severity information is entered into the model, the STM outputs a tailored triage strategy that prioritizes patients for transport to medical centers and directs casualties to particular facilities. The model is preconfigured with the capacity limitations of health care facilities in the surrounding region, and the model distributes victims among the nearby facilities based upon medical need and resource availability.

Survival estimates used to drive the triage strategy in the STM are evidence based, although these values are not derived from mass casualty incidents and the underlying data that the model was built upon remain unpublished. ${ }^{17}$ Under normal circumstances, trauma victims receive significantly more prompt and more extensive medical care than would be feasible in a mass casualty situation. Moreover, the STM also fails to consider the different amounts of time that are required to treat different types of injuries. Thus, the model may predict overly optimistic outcomes. Of particular importance, STM was not designed to consider either combined injury or the distribution and quantity of victims that would be expected after a nuclear detonation. In addition, robust methods that account for differences in patient survivability exist to prioritize the use of ventilators during a pandemic influenza outbreak ${ }^{18}$; however, this system is not applicable to victims of a nuclear incident.

\section{MORTT AS A MODEL FOR POPULATION-BASED SURGICAL TRIAGE AFTER A NUCLEAR DETONATION}

For the reasons mentioned above and because the STM model is not available to the public, we sought to develop a model to test different hospital-based triage approaches after a nuclear detonation. We call this model the model of time and resourcebased triage (MORTT). The purpose of developing MORTT was to guide the use of scarce medical resources, especially the time of critical hospital-based personnel, in the first day or two after the detonation of an improvised nuclear device (IND). MORTT is not intended to be used by the medical community in the aftermath of a disaster, but rather to be used as a tool to explore the effect of various prioritization decisions preevent to support planning in an environment in which medical resources are scarce.

MORTT focuses primarily on the surgical needs of trauma victims, because life-threatening manifestations of acute radiation syndrome typically begin several days after exposure in those who received survivable doses. ${ }^{6,19}$ The focus on trauma is underpinned by the notion that the casualties of a nuclear incident should be first triaged based on conventional injuries (eg, burns, blast injuries, other trauma) before considering potential radiation exposure. ${ }^{20} \mathrm{We}$ discuss below that MORTT considers additional mortality from combined injuries and can guide the prioritization of victims who received radiation in addition to trauma. We used this mathematical model to explore various triage schemes and determine their relative practical utility in the aftermath of a nuclear detonation, as described in the Results section. There are also triage considerations for radiation alone based on survivability and resource consumption, which are considered by DiCarlo et $\mathrm{al}^{6}$ and in the triage tool recommendations of Coleman and colleagues. ${ }^{?}$

Several limitations in MORTT exist (Table 1), some of which relate to any model, whereas others are specific to the available data and predictions that are germane to a nuclear detonation. These limitations are important to consider within the context of planning for individual health care/medical facilities and municipalities. Most important, addressing these limitations with new research will enhance the predictive value of 
MORTT, in turn enhancing the ability of the model to guide planning efforts.

\section{METHODS}

As described above, MORTT is designed to test various hypotheses related to the prioritization of victims after a nuclear detonation and the number of lives saved by that prioritization scheme. The model must reflect the medical resources needed initially to treat/stabilize the variety of types of injuries that are likely to present in the incident and the time to treat these victims. In addition, the model must account for the consequence of those resources not being available. Simply put, the model must predict the probability of additional mortality or morbidity if those resources are withheld. To be applicable to a particular incident, the model must be supplied with victim populations that reflect reality. In this section, we first describe how the model works and then discuss the data sets that inform the model.

\section{Structure of MORTT}

MORTT is embodied as a Microsoft Excel file with a simple underlying algebraic structure. The model is agent based, in that each "agent" is a medical team that chooses and then treats victims based on the available victims in the victim pool and the triage priorities entered by the user. If several injury types are simultaneously next in priority order (eg, all of the moderate injuries regardless of mechanism or location), then the model assigns a medical team to one of those victims based on a random draw from the total victims available across those injury types. Upon assignment to a patient, the medical team is occupied for the length of time required to treat an average patient in that injury type. After the patient is treated, the medical team is assigned a new patient from the current pool of untreated patients based on the assigned priority order. While waiting for care, a portion of victims dies as determined by a death rate specific to each injury type, as in the formula:

Untreated victims remaining $_{\mathrm{T} 1}=$ Untreated victims remaining $_{\mathrm{TO}}$ - (Untreated victims remaining TO $^{*}$ Death Rate * Time)

Even if treated, a victim may still die (because not all of the treatments are successful) based on the rate of mortality after treatment specific to each injury type. The model runs only for 48 hours, at which time any surviving victim (generally those with mild injuries only) is considered treated. There-

\section{TABLE 1}

\section{Limitations of MORTT and Consequences to the Model}

\section{Limitation}

No downtime between surgeries is included

Critical material resources are not included

Resources are aggregated across the region instead of allocated to particular hospitals

All patients arrive at the hospital at the same time

MORTT focuses only on surgeries

Untreated death rates are based on deaths recorded after battlefield injuries during transport to a field hospital

MORTT considers that victims are stabilized and transported for definitive treatment

Number of anesthesiologists and anesthetists limits the number of total surgical teams because these personnel are more limiting than surgeons or surgical nurses

Combined injury data are generated from animals who received non-life-threatening trauma in addition to radiation

Victims with non-life-threatening injuries are excluded from MORTT

Earthquake data were derived from areas with few collapsed high-rise buildings

No data exist on the benefit of treating traumatic injury on survival of victims with combined injury

\section{Consequence}

Resource availability is likely to be overly optimistic in the model; therefore higher victim-to-treater ratios should be used to compensate for this shortcoming; addressed in sensitivity analysis.

Although personnel resources are required to administer material resources, some material resources may be extremely limiting, preventing the effective treatment of some trauma victims

Local shortages and surpluses are likely to make the response more inefficient than modeled; therefore, higher victim-to-treater ratios should be used to compensate for this shortcoming; addressed in sensitivity analysis

Difficulties in patient transport may significantly limit the number of severely injured patients received by a hospital; addressed in sensitivity analysis

Activities outside the operating room will clearly have an impact on the stream of patients ready for surgery and could improve survival of patients waiting for surgery

These patients are under the care of a medic or corpsman (cannot truly be considered untreated), so death rates may be low; addressed in sensitivity analysis

Patient evacuation may be limited in the aftermath of an IND, limiting survival probability for victims of trauma

In a life-threatening emergency, perhaps some surgeries would be performed with anesthesia that require less supervision

It is unknown whether more severe trauma will further exacerbate mortality from radiation or will reduce the combined effect if the trauma is deadly on its own

Although these victims are excluded because they have no impact on the number of lives saved if left untreated, they will undoubtedly consume resources, thereby reducing the efficiency of the medical system as a whole; also, these victims are likely to consume long-term care resources but are not counted in MORTT

MORTT likely overestimates the survivability of victims in collapsed heavy buildings in urban areas after an IND

MORTT likely overestimates the mortality of victims with combined injuries treated for traumatic injury; however, sensitivity analysis shows that this overestimate will not affect our conclusions

IND=improvised nuclear device; MORTT = model of time and resource-based triage. 
fore, the number of surviving victims of each injury type is given as:

$$
\begin{gathered}
\text { Surviving Victims } 48 h=(\text { Untreated Victims } 48 h+ \\
\left.\sum \text { Treated Victims }\right) * \text { Treated Survival Rate }-\sum \text { Dead Victims }
\end{gathered}
$$

Note that, as this equation shows, at the end of the model, all of the surviving untreated victims are considered treated and survive or perish at that point based on the treated survival rate. In this way, those surviving to the end of the model run are not considered to survive automatically.

This model attempts to replicate the emergency care given in hospitals in historical mass casualty incidents, in which medical teams serially treat victims instead of attempting to treat many patients at once, as is common practice in day-to-day emergency care. For example, during the response to the Rhode Island nightclub fire in 2003, one physician reported that "there were at least 30 leaders, each gathered with a small team at the bedsides of the most critically ill patients. Once stabilized, each patient would be whisked away, and almost immediately another would be rolled in to take his or her place." ${ }^{21}$

The model starts with a population of untreated patients and as time advances, the patients are moved to other groups, such as dead, treated, or palliative care. Material resources needed to treat the victim are consumed, and when all of the necessary resources of that type are consumed, no more victims who require that resource can be treated.

The user inputs the number of medical teams available in MORTT, including injury-based constant death rates, which transfer untreated patients to the dead pool. Finally, if victims are left untreated for too long or if sufficient resources are not available to treat a patient, untreated patients can be moved to the palliative care group (where they consume resources for palliative care).

The patients awaiting treatment are described by injury location, severity, and mechanism. MORTT can accommodate a nearly unlimited number of victim types, but we found that 68 injury types were sufficient to represent the trauma that is common after a nuclear detonation. The data underpinning the parameters for each victim type (eg, death rate, probability of survival if treated, time to treat) are described below.

Given various patient priorities entered by the user, MORTT assigns resources and then calculates the total lives saved by the system for any given set of resources available. In this way, the user can test the relative efficacy of triage schemes given a victim stream. In this study, we used MORTT to determine the relative efficacy of prioritizing victims based on injury severity or combined injury status. The MORTT can be used to test any triage scheme, including prioritizing injury location (eg, limb vs abdomen injuries), injury mechanism (eg, blunt vs penetrat- ing), a special population (children), or other criteria of unclear importance (eg, age, comorbidity). We tested these criteria in a sensitivity analysis.

MORTT was built as a flexible platform to consider limitations on various personnel and material resources. In this project, we focused only on 2 resource types: the personnel required to perform stabilizing surgeries and the medications needed to provide palliation. We focused on surgeries because outcomes are universally poor if a critical surgery is not performed and if resources needed to stabilize a victim are in short supply. Shortages in other resources will certainly reduce the probability of favorable outcomes, but emergency surgery is absolutely essential for survival. In addition, many nonpersonnel resources could be substituted for others. For example, patients who need whole blood could be given plasma or intravenous fluids instead to partially mitigate a shortage of blood. Other resources, however, are difficult to substitute for surgical teams. Our primary data source has data on surgical resources for all patient codes (PCs), but inconsistently tracked other types of resource needs (especially material resources). We focused on the medications required for palliation because the lack of these medications implies that some victims would not be provided the resources needed to manage pain or nausea, which was considered an undesirable outcome in itself.

We used an extensive set of test data to verify that the model worked as predicted. That is, we tested the model with simple victim pools (at first, only 2 victim types at a time) to ensure that the victim prioritization, treatment, and survival modules were working properly. We then continued to increase the complexity of the input data (eventually getting to more than 50 victim types), ensuring that the outputs were consistent with expectations. Validation of the model would require the occurrence of a large disaster that challenges available resources (eg, earthquake) in a setting in which injury types of treated and deceased victims is accurately tracked alongside the resources available to treat the victims.

\section{Resources to Treat Trauma Victims}

Data applicable to a civilian medical system in the aftermath of a catastrophic disaster are extremely limited. Studies of the resources required to treat victims in a non-resources-constrained setting are likely to overestimate requirements during a mass casualty response because many victims in the latter scenarios will receive stabilizing, not definitive, care. In contrast, in the military, it is normal practice to use a staged treatment approach with stabilization of traumatic injuries in a forward-deployed medical center (a field hospital) before deployment to a rear-echelon facility for definitive care and convalescence. A rich data set, called the time task treater files (TTTFs) has been developed to predict the resource needs of military victims in each echelon of care. ${ }^{22}$

The TTTFs contain specific PCs for different combat-related injuries, and each PC lists a set of tasks required to treat that injury. Each task under a certain PC also includes the material required, the time to complete the task, the number of times 
TABLE 2

\begin{tabular}{llll}
\hline \multicolumn{2}{l}{ Parallels Between Military and Civilian Medicine } & & \\
\hline Military Level & Military Treater & Civilian Level & Civilian Treater \\
Level 1: battalion aid station & Medic/corpsman & Ambulance & Emergency medical technician \\
Level 2: field hospital & Combat surgeon & Emergency department/ operating room & Surgical team \\
Level 3: rear-echelon hospital & Surgeon/specialist & Surgery, post-op, recovery wards & Specialist
\end{tabular}

\section{TABLE 3}

\section{TTTF Statistics on Detailed Injury Severity}

$\begin{array}{lcc}\text { Injury Severity } & \text { No. PCs } & \text { Relative Time to Treat } \pm \text { SD } \\ \text { Non-life threatening } & 75 & \text { NA } \\ \text { Mild } & 5 & 1.0 \pm 0.5 \\ \text { Moderate } & 52 & 2.0 \pm 1.4 \\ \text { Severe } & 45 & 7.3 \pm 3.9\end{array}$

Mild, moderate, and severe indicate severity of life-threatening injury. All times are relative to the time to treat the average victim with a mild life-threatening injury. $\mathrm{PCs}=$ patient codes; $\mathrm{SD}=$ standard deviation; TTTF=time task treater file.

per day the task is typically applied, and the percentage of patients who require that task. From these data, the total amount of treatment time for various types of personnel can be calculated for each patient depending on the type of injury he or she received.

The TTTFs detail 435 possible injuries encountered during wartime and span the categories of wounded in action, nonbattle injuries, radiation, nerve agents, diseases, stress, drug abuse, and sensory failure. We narrowed down the total list to 177 PCs consisting of primarily wounded in action codes and a few others that were applicable (eg, PC311, Eye Wound Lacerated) to the injuries likely to be encountered in the aftermath of an IND (see eTable in Supplemental Information at www.dmphp .org). MORTT assigns each of the PCs to 1 of the 68 injury types (Supplemental eTable). As mentioned above, the TTTFs were attractive for this application because they explicitly list the activities required to stabilize various types of trauma victims before transport for definitive care. When comparing the system contemplated by the TTTFs, we find elegant parallels between military and civilian medical protocols (Table 2). In the TTTFs, surgeries required to stabilize a victim before transport are performed at the field hospital level, whereas recovery and follow-up surgeries are performed at rear-echelon locations. We focused on the field hospital level of care to determine the resources needed to treat victims in the immediate aftermath of an IND detonation.

Each victim type in MORTT is described by a body location, severity, and mechanism. The location parameters are head, thorax (within the rib cage), abdomen, upper limbs, lower limbs, burns, and multiple injuries. Severity of an injury was assigned to PCs and injury types by using the descriptive scoring guidelines in the Abbreviated Injury Score system. ${ }^{23}$ Here, mild in- juries correspond to a score of 1 to 2 , moderate injuries to a 3 to 4 (except for injuries to the head or spine, in which severe injuries receive a score of $\geq 4$ ), and severe injuries were assigned a score $>4$. Mild injuries were further divided into lifethreatening and non-life-threatening injuries (because only a few posed a short-term risk to life even if untreated). The mechanism of injury could either be blunt, penetrating, or crush injuries. We only focused on all of the tasks conducted after applying anesthesia, thus indicating surgery. Moreover, we did not include administration tasks such as DOCUMENTATION, tasks that appeared to be machine related such as SUCTION, MAINTAIN and $\mathrm{O}_{2}$ ADMINISTRATION, and nursing and maintenance tasks such as VENTILATOR SET UP and APPLY DRESSING. We also removed tasks that were applied to only $\leq 1 \%$ of the population.

Although, in general, injuries to the extremities show low rates of mortality in normal resource settings, the surgical procedures used to treat these injuries are relatively lengthy and consume considerable personnel resources in the TTTFs during level 2 care. To recapitulate medical care for limb injuries in the aftermath of a nuclear incident, we considered that tourniquets would be used instead to stabilize victims with vascular injuries to the extremities. The approach to estimating the resources for tourniquet use is described in the online-only supplemental information.

\section{Examining the TTTFs and Treatment Time}

Relative time to treat injuries based on severity category is outlined in Table 3. These data have several limitations, as outlined in Table 1. Most notably, the model includes only surgical time and not time for preparing between patients or time for surgeons to wash, reglove, eat, or sleep. For this reason, we feel that the baseline estimates for resource utilization are optimistic, as discussed further below.

\section{Rate of Death and Probability of Survival if Treated}

The literature is extremely sparse regarding the time to death for patients awaiting lifesaving care. A body of literature exists on the time to death of patients in a hospital after admission, but these sources are not relevant to the time to death of untreated patients. ${ }^{24-26} \mathrm{We}$ tried to develop untreated death rates for various injury types based on predicted blood loss, given that a decrease in blood volume of $60 \%$ is widely accepted to be incompatible with life. ${ }^{27}$ Although we found relationships for the rate of blood loss to various injury types, upon evaluation of our findings, we discovered that our time-to-death values were 
unrealistically short because this approach did not account for physiological mechanisms used by the human body during hemorrhage to decrease blood flow through damaged vasculature or for the ability of lacerations to clot and heal after injury.

Instead, we used the TTTFs to predict the probability of death if treated and the rate of death if untreated for various injury types. Specifically, there is a specific task entitled MORGUE CARE in the more severe PCs in the TTTFs. Morgue care tasks were assigned to a percentage of patients in each echelon of care, denoting that death occurred either before arrival at a field hospital, during surgery, or during convalescence. To obtain the probability of death if treated, we totaled the probabilities of death across all of the echelons of care for each injury type. To calculate the rate of death while not treated, data on the delay from injury to hospitalization were gathered for a number of recent conflicts, including the Vietnam War, the Gulf War, the Bosnian War, and Operation Iraqi Freedom. ${ }^{28-33}$ From these data, we concluded that the deaths recorded before victims' arrival at a field hospital were experienced in the first 70 minutes after injury. Each of these injuries and their probability of death during that time were aligned with a similar type of injury produced by the IND. Given that statistic, we can calculate a rate of death, which is applied to the surviving, untreated victim pool. In the military, during transport to a field hospital, the victim receives care from a corpsman or medic and cannot strictly be considered untreated; therefore, estimated death rates based on deaths before arrival at a field hospital are probably too low. For this reason, we performed sensitivity analyses to determine the effect of altered death rates on outcomes.

In the model, patients waiting for treatment die at the death rate for their injury type. Once treated, a probability of death for each victim is applied based on the sum of probabilities of death for level 2 (field hospital) and level 3 (rear-echelon hospital) care in the TTTFs. Thus, the outcomes depend on an intact evacuation and secondary hospital infrastructure, which is unlikely in the aftermath of a nuclear detonation. Total death rates and probability of death if treated are shown in Table 4. Probabilities of death for various injury severities as derived from the TTTFs closely mirrored death rates associated with various injury severities in civilian hospitals (Table 5). ${ }^{34,35}$

\section{Casualty Streams Generation}

To estimate trauma types and numbers of victims, we used models from Lawrence Livermore National Laboratories (LLNL) and Science Applications International Corp (SAIC). These models predict the heat flux, radiation dose (from prompt effects and fallout), and overpressure experienced by various segments of a city after a nuclear detonation. Although burns could be caused by the heat flux, urban structures shield victims from the heat of the blast, such that almost no burn casualties result from the flash apart from those on people killed by other effects of the blast.

For the purpose of this model, we used the example of a 10kiloton (kT) ground burst in a major American city. Overpressure outputs from these models generated casualties in two ways:
TABLE 4

$\begin{aligned} & \text { Predicted Death Rates and Probability of Death if } \\ & \text { Treated by Injury Severity for Life-Threatening Injuries }\end{aligned}$
$\begin{array}{lcc}\text { Severity } & \begin{array}{c}\text { Untreated Death Rate/70-Min } \\ \text { Interval } \pm \text { SD, \% }\end{array} & \begin{array}{c}\text { Treated Probability } \\ \text { of Death } \pm \text { SD, \% }\end{array} \\ \text { Mild } & <1 \pm 0.3 & \\ \text { Moderate } & 4 \pm 0.2 & 7 \pm 0.2 \\ \text { Severe } & 20 \pm 9 & 60.7 \\ \end{array}$

The death rate is suffered for every 70-min interval before treatment, so all of the untreated casualties will die if untreated. $S D=$ standard deviation.

\section{TABLE 5}

\begin{tabular}{|c|c|c|c|}
\hline & Mild & Moderate & Severe \\
\hline \multicolumn{4}{|l|}{ ISS } \\
\hline Blunt, \% & 0.1 & 8 & 57 \\
\hline Penetrating, \% & 0.1 & 6 & 65 \\
\hline AIS, \% & 0.5 & 8.5 & 59.5 \\
\hline TTTF, \% & 0.2 & 7 & 60 \\
\hline
\end{tabular}

AIS=Abbreviated Injury Scale; ISS=Injury Severity Scale; TTTF=time task treater file.

victims could be injured by flying glass from shattered windows and by building damage from the blast wave. Although many people are predicted to be exposed to flying glass from ruptured windows, these injuries are rarely life threatening because the glass tends not to pierce the body wall. ${ }^{36}$ Because these injuries are not life threatening, early treatment does not figure into lives saved in our model and is excluded. We also excluded trauma resulting from car crashes caused by the blast because of the great uncertainty of the number and severity of injury of these victims. Thus, the primary driver of trauma in the LLNL and SAIC models is the structural failure of buildings, which are much more sensitive to overpressure than are body structures. ${ }^{5}$ As predicted by Glasstone and Dolan, ${ }^{37}$ building failure was forecast as a function of overpressure.

Although the exact casualty predictions resulting from the detonation of an IND in a major US city are not appropriate to publish in a public journal, some approximate figures can provide context. These models predict that tens of thousands of victims will receive life-threatening injuries, which, if the medical system were completely intact and not overwhelmed, would have a good chance of survival. About twice as many people are predicted to experience moderate trauma than those who undergo severe trauma. In addition, many tens of thousands will experience trauma so severe that they never reach a hospital. Many more people will receive relatively minor injuries that normally would be treated in a hospital, but because of the unprecedented surge of victims, their care is likely to be limited to basic first aid in the days after the disaster. 
TABLE 6

\section{Injury and Death Distribution From Building Types for Life-Threatening Injuries and Promptly Dead ${ }^{38-49}$}

\begin{tabular}{lcc} 
Injury Severity & Heavy Building, \% & Light Building, \% \\
Mild & 29.36 & 41.77 \\
Moderate & 32.64 & 22.34 \\
Severe & 8.1 & 21.65 \\
Promptly dead & 29.9 & 14.24 \\
\hline
\end{tabular}

\section{TABLE 7}

\begin{tabular}{|c|c|c|c|}
\hline \multirow[b]{2}{*}{ Type } & \multirow[b]{2}{*}{ Subtype } & \multicolumn{2}{|c|}{ Medical Professionals } \\
\hline & & $\begin{array}{c}\text { In Wasington, } \\
\text { DC }\end{array}$ & $\begin{array}{c}\text { In DC Metro } \\
\text { Area }\end{array}$ \\
\hline \multirow[t]{5}{*}{ Doctors } & Total physicians & 1535 & 12300 \\
\hline & $\begin{array}{l}\text { Surgeons: general and } \\
\text { orthopedic }\end{array}$ & 182 & 1500 \\
\hline & Surgeons: other & 343 & 2700 \\
\hline & Anesthesiologists & 54 & 400 \\
\hline & $\begin{array}{l}\text { Emergency medicine } \\
\text { physicians }\end{array}$ & 59 & 500 \\
\hline \multirow[t]{3}{*}{ Nurses } & Total nurses & 6510 & 52100 \\
\hline & $\begin{array}{l}\text { Critical care and operative } \\
\text { nurses }\end{array}$ & 644 & 5200 \\
\hline & Nurse anesthetists & 70 & 600 \\
\hline
\end{tabular}

Using Data From Earthquakes to Populate the Victim Pool Given that the majority of life-threatening trauma produced by an IND is likely to arise from people caught in or near buildings that are destroyed or heavily damaged by the blast wave, we searched for sources of data that detailed the types of injury from this cause. At first, we considered the data on explosions that collapse buildings; however, many victims of explosions are hurt by the overpressure wave itself, causing damage to the lungs and soft tissues of the abdomen. Because victims who have a chance of surviving a nuclear detonation are unlikely to undergo this type of direct blast damage, we instead focused on studies of trauma caused by buildings damaged in earthquakes. From this literature (references in Table 6), we collected data on trauma resulting from crumbling buildings based on building type, body location of the injury, injury severity, and injury mechanism (ie, penetrating, crush, blunt injuries).

We condensed all of the building types data into categories of either heavy (eg, reinforced concrete structures, high-rise multifamily dwellings) or light (eg, single-family homes, other woodframe structures) structures. Percentage of people injured, severity of the injury, and number of promptly dead from the two building types are presented in Table 6 . Using data provided by SAIC that indicated how many people were in heavily damaged buildings after an IND and whether these buildings were heavy or light, we determined the total number of trauma victims and the severity of their injuries. People considered promptly dead were excluded. We note that urban centers have many more skyscrapers than the locations from which the earthquake data was derived; therefore, MORTT possibly overestimates the survivorship of victims in high-rise buildings.

In a parallel effort, we determined the overall severity, body location, and mechanism of all of the injuries resulting from damaged buildings (data not shown). These data were entered into a final injury distribution model, which takes the number of people in heavy-and light-structured buildings that have been heavily damaged and distributes the injuries of the victims accordingly. If an injury category/severity combination resulted in $<5 \%$ of the total population, then that combination was removed to reduce the computational complexity of the model.

\section{Available Resources Estimation Personnel Resources}

The number of surgical teams dictates the number of independent "agents" in the model, each of whom chooses a patient from the highest-priority group, treats the patient, and is released when finished. A typical surgical trauma team has 2 surgeons, 3 nurses, 2 physicians, and 1 anesthesiologist. ${ }^{50-56} \mathrm{~A}$ full discussion of how we determined which type of personnel was most limiting to set the baseline number of surgical teams is found in the online-only supplemental information. In the aftermath of a large disaster, it may be questionable whether all of these personnel would be used to treat each victim. However, the data on time-to-treat exists only for trauma teams as they exist today, and paring down the personnel would probably increase the time needed to treat any injury and therefore have limited effect on the model. Also, there are so many uncertainties about the availability of medical personnel in the aftermath of a nuclear incident that the composition of the team is a relatively minor concern. Furthermore, it was unclear how much down-time these teams would be afforded between victims, which can significantly reduce the resources considered available by the model. Because of the uncertainty in this parameter, we used MORTT to test how the availability of fewer and more surgical teams affected the results. The sensitivity analysis also accounts for variability in the composition of the trauma teams (if the same treatments could be completed as quickly by a smaller team than the teams used normally, then the smaller ratios of victims to treatment teams is closer to the truth). Even if our assumptions are significantly inaccurate, the conclusions of the present article hold, and as resources become more constrained, the importance of the suggested triage order increases.

\section{Resources for Palliation}

As described above, if a patient dies untreated, then they are considered to have been shifted to palliative care. Although no additional deaths occur if resources for palliation are lacking, suffering without palliation is a poor outcome. Therefore, we estimated the extent of resources in the Washington, DC, metropolitan area potentially available for palliative care, as described in Table 7 and the online-only supplemental material. 


\section{RESULTS}

\section{Lives Saved by Triage}

We used MORTT to explore the following hypothesis: In the aftermath of an IND, triage strategies that prioritize less severely injured victims over more severely injured victims will save more lives. We based this hypothesis on expectations that resource and time limitations will be widespread and severe, and the finding that more severely injured victims would require greater time and resource allocations per life saved than less severely injured victims (Table 3). To test the hypothesis, we loaded MORTT with the victim populations and data on available surgical teams, as described above. We ran the model to represent the first 48 hours of the response postdetonation. A major limitation (Table 1) was that we assumed that all of the victims who were treated could be transported elsewhere for definitive care, which is highly uncertain in the aftermath of a nuclear detonation.

We understand that there is great uncertainty regarding how many patients and how many surgical teams will present to the hospital system; thus we reran the model several times using varying ratios of victims to surgical teams compared with baseline (Figure 1). We present the results as the relative number of lives saved compared with a conventional triage system, in which severely injured victims are prioritized. For reference, we also present the results of a system in which the only form of triage is to delay victims with nonfatal injuries until all life-threatening injuries are treated. There is little run-to-run variability in MORTT simply because the large numbers of surgical teams and victims result in minimal run-to-run variability (standard deviation for these results is approximately $1 \%$ ).

Multiple findings are notable. First, when the victim loading is low (ie, less than or equal to the baseline number of surgical teams and patients, called " $1 \mathrm{x}$ " in Figure 1), a triage system that prioritizes moderately injured victims followed by severely injured victims followed by mildly injured victims (mod-sev-mild) saves $10 \%$ more lives than alternative approaches. Second, as the victim loading increases relative to the resources available (up to 10 -fold more patients or 10 fold fewer surgical teams as the baseline, called " $10 x$ " in Figure 1), mod-sev-mild saves more than 3-fold more victims than a sev-modmild system. In contrast, a system that prioritizes mildly injured victims (who have a long time to death) results in comparatively fewer lives saved as victim loading increases. The relatively good performance of "no triage" results from the preponderance of moderately injured victims in the overall victim pool (more than two-thirds of victims with life-threatening injuries are "moderate"), such that a strategy with no triage will commonly select moderately injured victims. As discussed above and outlined by DiCarlo and colleagues, ${ }^{6}$ higher levels of victim loading may better reflect the performance of the medical system aftermath of a nuclear detonation.

The data available to estimate both casualty streams and the performance of the medical system in the aftermath of an IND are fraught with uncertainty (Table 1). To address this uncertainty, we performed an uncertainty analysis to determine the effects from changing the underlying data on outcomes. The initial analysis assumes that all of the victims arrive at the hospital and enter a victim queue

\section{FIGURE 1}

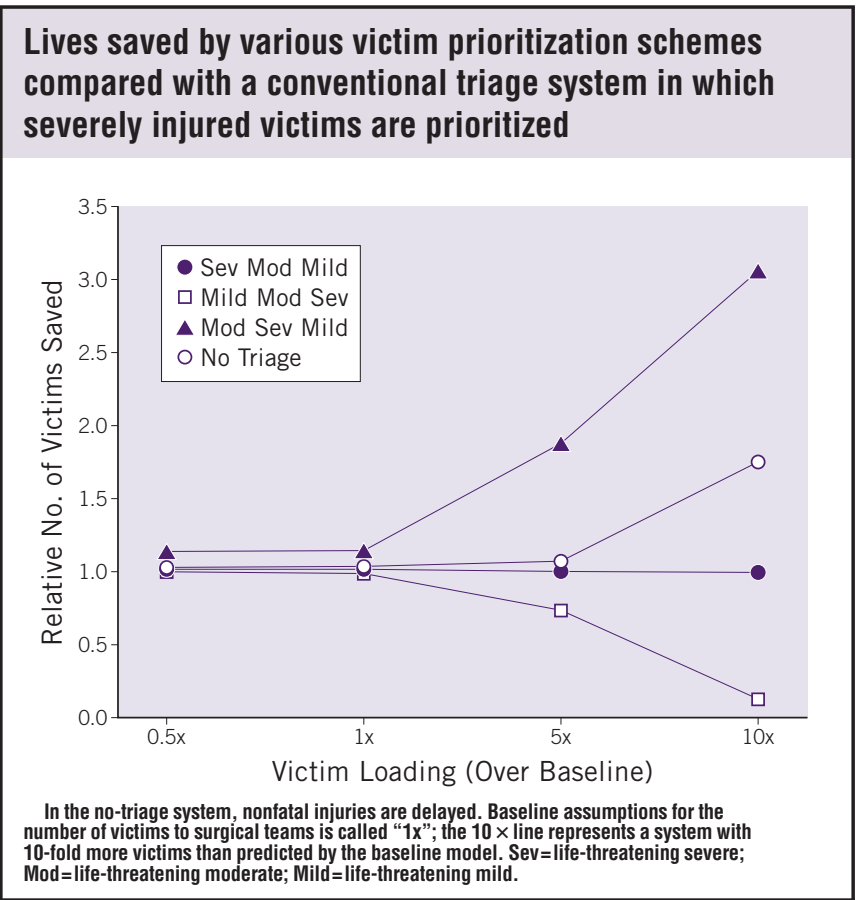

FIGURE 2

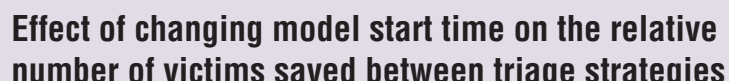
number of victims saved between triage strategies

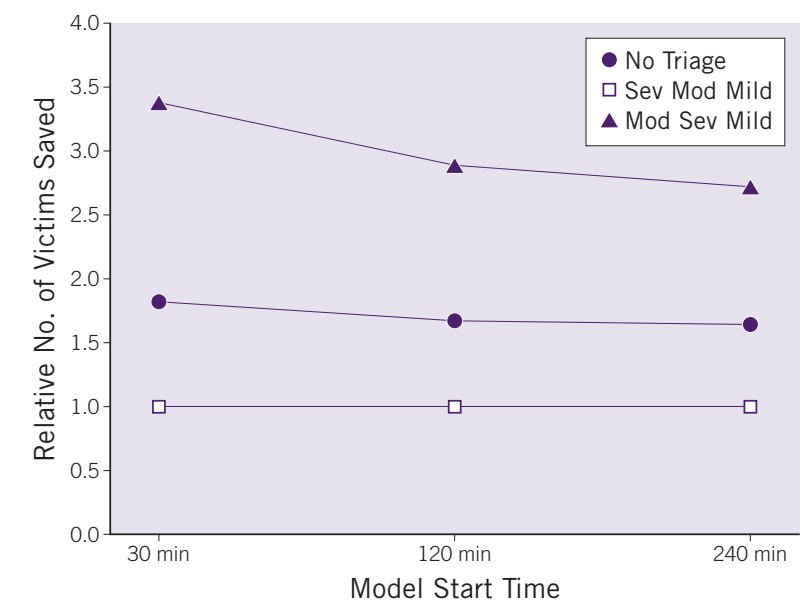

70 minutes after the incident (based on figures from the transport of military victims). Difficulties in the transportation of victims within a targeted city and the recommendation to shelter in place may, however, significantly delay the presentation of victims to the hospital. The change in "start time" for MORTT affects the composition of the victim pool in that later start times have proportionally fewer severely injured victims in the victim pool simply because these victims die more quickly than moderately or mildly injured victims. At $10 \times$ victim loading, the relative benefit of mod-sev- 


\section{FIGURE 3}

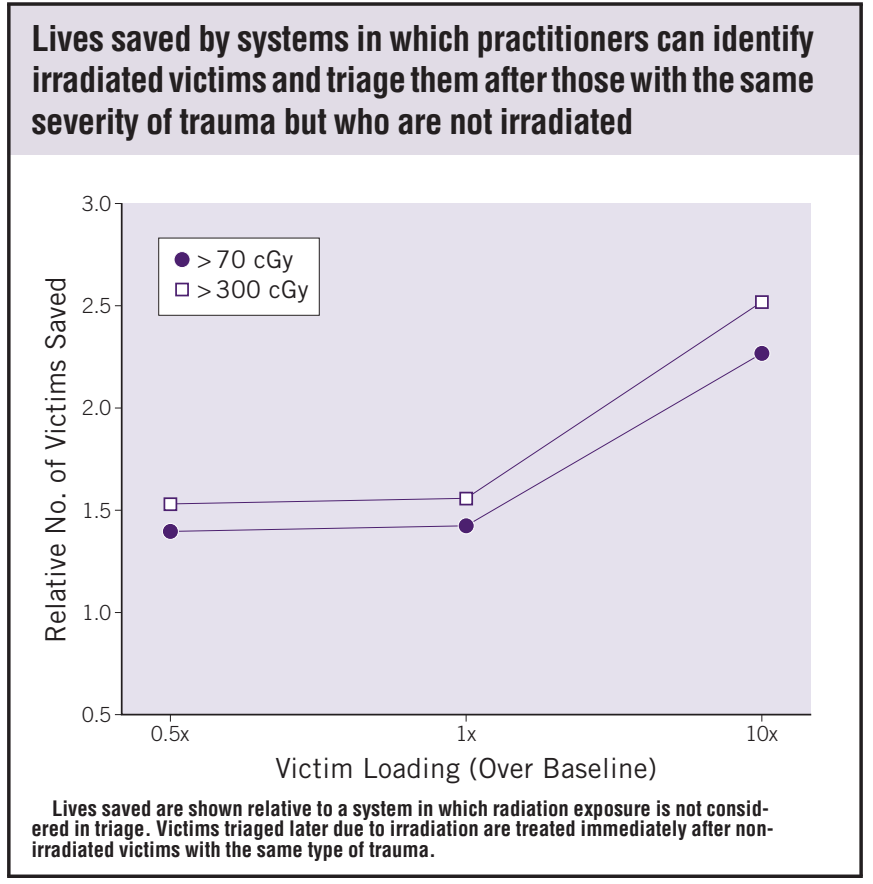

mild oversev-mod-mild is inversely proportional to model start time, but varies only slightly over the range of 30 to 240 minutes (Figure 2).

Although the rates of death from TTTFs replicate the frequency of death observed in civilian hospitals (Table 5), we tested the effects of higher death rates. Higher death rates could occur if postsurgical care is compromised, surgical resources are more limited than predicted, or the nuclear detonation occurred during inclement weather, when a lack of adequate shelter could compromise the ability of patients to survive. We therefore increased the rate of death by $5 \%, 10 \%$, or $20 \%$, or doubled the death rate for each category. In every case, the mod-sevmild triage strategy saved the most lives (data not shown). For example, if the death rate is doubled for each category, then a triage system that prioritizes the moderately injured saves $40 \%$ more lives with $1 \times$ victim loading than a conventional triage system, compared with $10 \%$ if the death rate is not doubled.

The TTTFs may not be applicable (in terms of time-to-treat and mortality) to the very young and the very old because the data are based on military age populations. To compensate, we researched the probability of death from trauma for pediatric and geriatric patients and data on time to treat and included these populations in our model. Across all of the age populations, the benefit of prioritizing or deprioritizing these groups was insignificant because they represented a small percentage of the victims with any type of injury (data not shown) in a nuclear detonation incident. Given the ethical issues associated with such a decision, we cannot suggest that any preference be given to a victim based on age.

\section{Combined Injury}

In the aftermath of a nuclear detonation, many victims with traumatic injury will also have been exposed to significant levels of radiation, either from prompt radiation or fallout. During the hours and days postdetonation, it may not be possible to distinguish which victims received significant radiation doses $^{6,57}$; however, new biodosimetry tools may make this distinction possible in real time. Thus, we explored whether approaches to combined injury could be modeled with MORTT.

As described above, we received data from LLNL and SAIC on the effects of an IND blast in a major city that included the radiation dose caused by prompt effects of the blast and from fallout. SAIC provided us with radiation dose data (modified for shielding and dose protraction for fallout) and overpressure experienced by a city's population to allow for calculating the number of combined injuries that would result from a nuclear detonation. The approach to determining the additional mortality associated with combined injury (compared with simple trauma) is described in the online-only supplemental material.

We used MORTT to determine whether triage of victims with combined injury after those with the same type of trauma but no radiation saves lives. We found that delaying the care of victims with trauma and $>0.7$ Gy of irradiation increases the number of lives saved by 1.4-fold compared to a system in which irradiated victims are treated exactly like nonexposed individuals (Figure 3). Moreover, the importance of radiation triage increases as victim loading increases. This type of radiation-based triage may be possible because those who received $\geq 0.7$ Gy may be experiencing prodromal symptoms such as nausea or vomiting. Alternatively, using a threshold of 3 Gy further increases lives saved compared with 0.7 Gy, reflecting the fact that doses between 0.7 and 3 Gy have less effect on overall survival, even when combined with trauma. Distinguishing those who received 3 Gy in a mass casualty setting will be difficult, supporting the need for new technologies to enable this type of careful discrimination.

We confirmed that delaying victims with combined injury saves lives across a range of ratios of victims to surgical teams (data not shown). In choosing the radiation dose threshold for deprioritization, we used the $>2$ Gy recommended in DiCarlo et $\mathrm{al}^{6}$ and Coleman et al. ${ }^{7}$ This is approximately 0.6 LD 50 (see eFigure in Supplemental Information at www.dmphp.org), which has a $30 \%$ to $50 \%$ decrement in survival when combined with non-life-threatening trauma and is also the threshold for use of antineutropenics for radiation alone. Recognizing the uncertainties in these data, using 2 Gy as a cutoff for both radiationalone treatment and for deprioritization with combined injury will make treatment less complex for responders. Furthermore, sensitivity analysis shows that even if the survival decrement from combined injury is less significant than we predict (which may be the case if open wounds are closed by medical intervention), then the benefit of the delay of those with combined injury after those with simple trauma of the same type 


\section{Demands on personnel in the intensive care unit (left) or in medical/surgical units (right) after a nuclear detonation based on triage scheme}

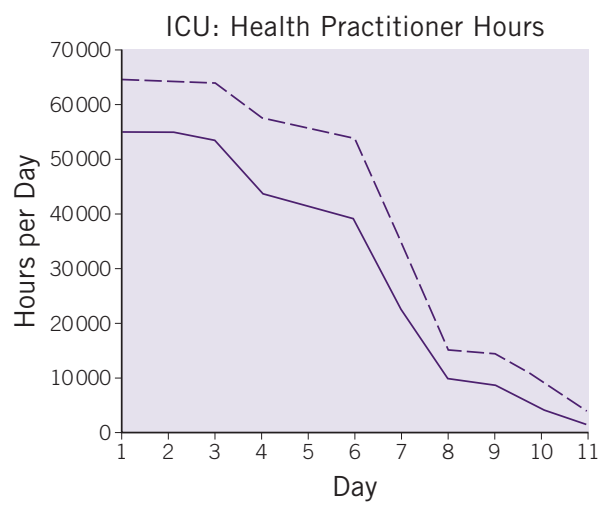

still saves more lives, although fewer additional lives are saved as the difference in survival decreases.

\section{Subsequent Health Needs}

By selecting which injury types to prioritize, a triage system approach will result in different surviving populations with specific needs for subsequent care. If subsequent care is unavailable, then any benefit from hospital-based interventions will be compromised. Thus, an optimal triage system would incorporate the subsequent care needs of victims as a secondary metric and the ability to provide this care; however, the availability of resources during a nationwide evacuation is extremely difficult to predict. ${ }^{6}$ Thus, the current modeling does not incorporate subsequent care needs into triage. Instead, the model was used to assess the impact of various triage orders on the need for subsequent care. The method for calculating the resources available for subsequent health needs (including floor beds and personnel, intensive care unit (ICU) beds, and personnel and capacity in nursing facilities and rehabilitation centers) is provided in the online-only supplemental material.

To determine the need for medical personnel to support recovery needs we turned to the TTTFs. We considered convalescent care to occur after all surgery in level 3 care, which is described earlier in the article. For each PC, we determined the average amount of time per day required in the ICU and the hospital floor, and averaged the total for each PC to determine the health care practitioner time required for each injury type and severity (as shown in the online-only supplemental material). This total reflects the care needed from the time that a patient enters the ward to the time that the patient is transferred from the ward or discharged.

Using these data, we calculated the demands on ICU and hospital floor resources for the surviving victims. We then applied these relations to the victims who survived treatment based on outputs from MORTT. The model predicts that a mod-sevmild triage scheme results in less demand for ICU beds than a sev-mod-mild scheme ( 15000 vs 17000 on the first day), even though the former scheme saves more lives overall. Given that only 9000 ICU beds are available without emergency measures taken, the demand for ICU beds will challenge the national capacity. Thus, any strategy to reduce ICU needs while saving lives is desirable. The reduced ICU needs from ModSev-Mild triage is primarily due to fewer survivors with thoracic and abdominal trauma and more survivors with serious injuries to their limbs. As expected, the demand on ICU personnel is also less for Mod-Sev-Mild, and does not differ across a range of victim loading (Figure 4).

In contrast, because some moderately injured victims can convalesce outside the ICU, the demands for care on medical/ surgical units are initially greater for the mod-sev-mild strategy compared with sev-mod-mild strategy (Figure 4). However, as victims are transferred from the ICU, the demands on medical/ surgical floors increase and last longer for triage schemes that prioritize severely injured victims. In either case, these demands on the hospital floor are significantly fewer than the resources available nationwide, suggesting that with effective triage and transport, essentially all of the victims who require non-ICU hospitalization could receive care in an appropriate setting. If, however, non-critical care beds are converted into ICU beds to address a shortage in critical care capacity, a shortage in floor beds may also be experienced. It should be noted that because MORTT focuses on medical needs in the immediate aftermath of the incident and on resources for care of traumatic injury, we did not consider the demand on ICU and floor beds that victims of acute radiation sickness will create in the days and weeks after the incident. If this additional demand were considered, then ICU resources would be even more scarce and floor space would become critical, reaffirming that the impact of triage schemes extends to resources beyond the first few days. 
Some patients discharged from the hospital may require additional care in nursing facilities or outpatient rehabilitation centers. We did not include psychiatric hospitals because they receive $\leq 3 \%$ of patients from any injury type, including head injuries. ${ }^{58} \mathrm{We}$ predict that demand on nursing facilities will not be a critical issue because even optimistic assumptions for resource availability result in the demand for fewer than 1000 nursing facility beds for any triage scheme after people with lifethreatening injuries convalesce, which is dwarfed by the national capacity. However, the model excludes those without lifethreatening injuries, who may require care in a nursing facility.

Outpatient rehabilitation is also variable by injury type, but many more patients will require outpatient rehabilitation than residence in a nursing facility. Almost one-third of all severely injured patients and some with moderate injuries will require rehabilitation, ${ }^{58-60}$ and the number of days spent in rehabilitation can range from 20 to 47 days. ${ }^{61,62}$ When considering the need for rehabilitation, we find that only 2000 victims are likely to need rehabilitation services to recover from life-threatening injuries, which is far below the available resources nationwide. The model excludes those who have received injuries to the eyes without additional life-threatening trauma. These patients are likely to need significant rehabilitation resources. $\mathrm{Nev}$ ertheless, triage schemes that prioritize severely injured victims result in a $10 \%$ greater demand for rehabilitation services compared with schemes that prioritize moderately injured people.

Traumatically injured patients return to the hospital more often in the 10 years after their injury than their uninjured counterparts, based on the 21000 patients enrolled in the Manitoba Injury Outcome Study ${ }^{63}$ We used these data to estimate the additional long-term hospitalization needs of moderately and severely injured patients. We find that the mod-sev-mild strategy results in a long-term care demand resulting from rehospitalization about $30 \%$ greater than the Sev-Mod-Mild scheme (30000 vs 20000 extra days). This finding presumably resulted from two facts: first, that the mod-sev-mild scheme saves more lives, and second, that rehospitalization rates are greatest for people with significant limb injures, which are a common injury type in the moderate injury group.

\section{CONCLUSIONS}

Using MORTT we determined that a mod-sev-mild triage strategy saves more lives than treating severely injured victims first. This guidance holds for various assumptions of resource demand and scarcity, transport time to hospitals, and death rates. Therefore, our conclusions hold in the face of the significant uncertainty faced when modeling nuclear incidents and the medical system. These results differ significantly from conventional triage schemes, in which the salvageable victim most likely to die next is prioritized, but these results are logical in the aftermath of a nuclear detonation for multiple reasons. First, severely injured people have a lower probability of survival even if treated. Second, severely injured people require more resources. Finally, medical resources may be unavailable to sta- bilize moderately injured victims while severely injured people are treated, and therefore people with moderate injuries will progress to a more severe category.

Also using MORTT, we determined that identifying trauma victims who received a significant dose of radiation could save additional lives by prioritizing the care of those with trauma alone, who are more likely to benefit from treatment, before those with significant combined injury. Tools that can identify and accurately diagnose people who have received significant radiation doses (eg, at least into major dose groups of $>2$ Gy prompt dose equivalent) will enable a more robust triage system and save more lives than a system that simply delays the treatment of anyone with possible combined injury.

We designed MORTT as a flexible framework for testing various hypotheses related to the allocation of limiting medical resources. MORTT is not intended to be used by the medical community in the aftermath of a disaster, but instead to be used as a tool to explore the effect of various prioritization decisions preevent to support planning in a scarce resources setting. In this study, we applied MORTT to predicted patient streams resulting from a nuclear detonation and tested various patient prioritization schemes based solely on a resource that we considered to be most critically limiting - the availability of surgical teams.

The MORTT platform enables users to determine how limiting resources would affect patient outcome by tracking the use and limiting the availability of any material or personnel resource in the model. The MORTT platform is broadly applicable to other disaster scenarios as long as the user can accurately predict the types and severities of injuries. To provide accurate predictions, data must be available for resource availability, injury-specific resource requirements, and the consequence of resource unavailability. Also, the ability of other resources to substitute for the desired resource and the consequence of providing partial treatments to patients should be considered. That is, for trauma patients that require surgery, the consequence of no surgical care is relatively easy to predict. Less clear are the consequences of an inadequate supply of blood products or substitution of crystalloid for plasma.

Clearly, more research is needed to better refine estimates of casualties, resource requirements, and availability of personnel and materiel (Table 1). MORTT could be greatly improved by the collection of primary data on the time to treat various types of patients in emergency situations in US nonmilitary hospitals (like multiple-vehicle crashes). A careful study of the delay between surgery and survivability of various types of trauma (from rural car crashes) or from other disasters would help inform the relations used to predict the consequence of delayed care. Moreover, more data on combined injury is needed, specifically related to the additional mortality experienced from combined injuries if the wounds are treated in the hours after they are inflicted compared with untreated combined trauma cases and compared with those with simple irradiation. Finally, MORTT represents an initial ef- 
fort to model a vastly complex event. We seek input from the emergency response and radiation/nuclear communities to improve its predictive value, both through better data to support the models and better study design to address the most important questions.

Author Affiliations: Dr Casagrande, Mr Wills, Ms Kramer, Ms Sumner, Mr Mussante, Ms Kurinsky, and Mr McGhee are with Gryphon Scientific; Dr Katz is with Abt Associates; Dr Weinstock is with the Dana-Farber Cancer Institute, Harvard Medical School; and Dr Coleman is with the Office of the Assistant Secretary for Preparedness and Response, US Department of Health and Human Services.

Correspondence: Address correspondence and reprint requests to Dr Rocco Casagrande, Gryphon Scientific, 6930 Carroll Ave, Suite 810, Takoma Park, MD 20912 (e-mail: rocco@gryphonscientific.com).

Received for publication September 19, 2010; accepted January 12, 2011.

The US Department of Health and Human Services (DHHS) provided funding to support this publication and convene the authors. The contents of the articles represent the personal views of the individual authors and do not necessarily express the opinion or policy of DHHS or its components. No statement in the articles should be construed as an official position of DHHS or its components. This work was also supported by the Agency for Healthcare Research and Quality, Department of Health and Human Services, and Gryphon Scientific.

Author Disclosures: The authors report no conflicts of interest.

Acknowledgments: The authors acknowledge the contribution of Alicia Livinski, biomedical librarian, National Institutes of Health Library, and Paula MurrainHill, program analyst, Office of the Assistant Secretary for Preparedness and Response, DHHS, for their assistance with the preparation of this manuscript. We thank Brooke Buddemeier of Lawrence Livermore National Laboratory and Richard Mcnally of Science Applications International Corp for their assistance with the models to estimate trauma types and numbers of victims.

\section{REFERENCES}

1. Iserson KV, Moskop JC. Triage in medicine, part I: Concept, history, and types. Ann Emerg Med. 2007;49(3):275-281.

2. Albin SL, Wassertheil-Smoller S, Jacobson S, Bell B. Evaluation of emergency room triage performed by nurses. Am J Public Health. 1975;65(10): 1063-1068.

3. Agency for Healthcare Research and Quality. Mass Medical Care With Scarce Resources: A Community Planning Guide. AHRQ Publication No. 070001. http://www.ahrq.gov/research/mce. Published February 2007. Accessed February 4, 2011.

4. Hick JL, Weinstock DM, Coleman CN, et al. Healthcare system planning for and response to a nuclear detonation. Disaster Med Public Health Prep. 2011;5(Suppl 1):S73-S88.

5. Knebel AR, Coleman CN, Cliffer KD, et al. Allocation of scarce resources after a nuclear detonation: setting the context. Disaster Med Public Health Prep. 2011;5(Suppl 1):S20-S31.

6. DiCarlo AL, Maher C, Hick JL, et al. Radiation injury after a nuclear detonation: medical consequences and the need for scarce resources allocation. Disaster Med Public Health Prep. 2011;5(Suppl 1):S32-S44.

7. Coleman CN, Weinstock DM, Casagrande R, et al. Triage and treatment tools for use in a scarce resources-crisis standards of care setting after a nuclear detonation. Disaster Med Public Health Prep. 2011; 5(Suppl 1):S111-S121.

8. Coleman CN, Knebel AR, Hick JL, et al. Scarce resources for nuclear detonation: project overview and challenges. Disaster Med Public Health Prep. 2011;5(Suppl 1):S13-S19.

9. Benson M, Koenig KL, Schultz CH. Disaster triage: START, then SAVEa new method of dynamic triage for victims of a catastrophic earthquake. Prehosp Disaster Med. 1996;11(2):117-124.

10. Romig L. The jumpSTART pediatric MCI triage tool and other pediatric disaster and emergency medicine resources. http://www.jumpstarttriage .com/JumpSTART_and_MCI_Triage.php. Accessed June 5, 2009.

11. Cook L. The World Trade Center attack. The paramedic response: an insider's view. Crit Care. 2001;5(6):301-303.

12. Hines S, Payne A, Edmondson J, et al. Bombs under London. The EMS response plan that worked. JEMS. 2005;30:58-60, 62, 64-57.

13. Tran MD, Garner AA, Morrison I, Sharley PH, Griggs WM, Xavier C. The Bali bombing: civilian aeromedical evacuation. Med J Aust. 2003; 179(7):353-356.

14. Jenkins JL, McCarthy ML, Sauer LM, et al. Mass-casualty triage: time for an evidence-based approach. Prehosp Disaster Med. 2008;23(1):3-8.

15. Garner A, Lee A, Harrison K, Schultz CH. Comparative analysis of multiplecasualty incident triage algorithms. Ann Emerg Med. 2001;38(5):541548.

16. Sacco WJ, Navin DM, Fiedler KE, Waddell RK II, Long WB, Buckman RF Jr. Precise formulation and evidence-based application of resourceconstrained triage. Acad Emerg Med. 2005;12(8):759-770.

17. Sacco WJ, Navin DM, Waddell RK II, Fiedler KE, Long WB, Buckman RF Jr. A new resource-constrained triage method applied to victims of penetrating injury. J Trauma. 2007;63(2):316-325.

18. Christian MD, Hawryluck L, Wax RS, et al. Development of a triage protocol for critical care during an influenza pandemic. CMAJ. 2006;175 (11):1377-1381.

19. Berger M, Leonard R, Ricks R, Wiley AL, Lowry PC, Flynn DF. Hospital Triage in the First 24 Hours After a Nuclear or Radiological Disaster. http: //orise.orau.gov/files/reacts/triage.pdf. Accessed February 4, 2011.

20. Kumar P, Jagetia GC. A review of triage and management of burns victims following a nuclear disaster. Burns. 1994;20(5):397-402.

21. Dacey MJ. Tragedy and response- the Rhode Island nightclub fire. N Engl J Med. 2003;349(21):1990-1992.

22. Defense Medical Standardization Board, US Department of Defense. The Defense Medical Materiel Program Office (DMMPO) Web site. https: //www.dmsb.mil/index.cfm. Accessed May 15, 2009.

23. The Abbreviated Injury Scale-1990 Revision (AIS-90). Des Plains, IL: Association for the Advancement of Automotive Medicine, Committee on Injury Scaling; 1990.

24. Acosta JA, Yang JC, Winchell RJ, et al. Lethal injuries and time to death in a level I trauma center. J Am Coll Surg. 1998;186(5):528-533.

25. Demetriades D, Kimbrell B, Salim A, et al. Trauma deaths in a mature urban trauma system: is "trimodal" distribution a valid concept? J Am Coll Surg. 2005;201(3):343-348.

26. Demetriades D, Murray J, Charalambides K, et al. Trauma fatalities: time and location of hospital deaths. J Am Coll Surg. 2004;198(1):20-26.

27. Alam HB, Shuja F, Butt MU, et al. Surviving blood loss without blood transfusion in a swine poly-trauma model. Surgery. 2009;146(2):325333.

28. Chambers LW, Rhee P, Baker BC, et al. Initial experience of US Marine Corps forward resuscitative surgical system during Operation Iraqi Freedom. Arch Surg. 2005;140(1):26-32.

29. Grau LW, Jorgensen WA. Handling the wounded in a counter-guerrilla war: the Soviet/Russian experience in Afghanistan and Chechnya. US Army Med Dept J. 1998;1/2:2-10.

30. Levitsky S, James PM, Anderson RW, Hardaway RM III. Vascular trauma in Vietnam battle casualties: an analysis of 55 consecutive cases. Ann Surg. $1968 ; 168(5): 831-836$.

31. Burkle FM Jr, Newland C, Orebaugh S, Blood CG. Emergency medicine in the Persian Gulf War-Part 2. Triage methodology and lessons learned. Ann Emerg Med. 1994;23(4):748-754.

32. Jevtić M, Petrović M, Ignjatović D, et al. Treatment of wounded in the combat zone. J Trauma. 1996;40(3)(Suppl):S173-S176.

33. Brethauer SA, Chao A, Chambers LW, et al. Invasion vs insurgency: US Navy/Marine Corps forward surgical care during Operation Iraqi Freedom. Arch Surg. 2008;143(6):564-569.

34. Civil ID, Schwab CW. The Abbreviated Injury Scale, 1985 revision: a condensed chart for clinical use. J Trauma. 1988;28(1):87-90.

35. Gennarelli TA, Wodzin E. AIS 2005: a contemporary injury scale. Injury. 2006;37(12):1083-1091. 
36. Bogosian D, Avanessian HD. Blunt trauma from blast-induced building debris. Paper presented at: 31st Explosives Safety Seminar; August 2004; San Antonio, TX.

37. Glasstone S, Dolan PJ. The Effects of Nuclear Weapons. 3rd ed. Washington, DC: Department of Defense; 1977:653.

38. Alexander D. Death and injury in earthquakes. Disasters. 1985;9(1):5760.

39. De Bruycker M, Greco D, Lechat MF, Annino I, De Ruggiero N, Triassi M. The 1980 earthquake in Southern Italy - morbidity and mortality. Int J Epidemiol. 1985;14(1):113-117.

40. Champion HR, Copes WS, Sacco WJ, et al. The Major Trauma Outcome Study: establishing national norms for trauma care. J Trauma. 1990; 30(11):1356-1365.

41. Coburn AW, Spence RJS, Pomonis A. Factors determining human casualty levels in earthquakes: mortality prediction in building collapse. Paper presented at: 10th World Conference Earthquake Engineering; 1992; Madrid, Spain.

42. Hare SS, Goddard I, Ward P, Naraghi A, Dick EA. The radiological management of bomb blast injury. Clin Radiol. 2007;62(1):1-9.

43. Jones NP, Wagner RM, Smith GS. Injuries and building data pertinent to the Loma Prieta earthquake: county of Santa Cruz. Monograph \#5. Paper presented at: National Earthquake Conference; May 2-5, 1993; Memphis, TN.

44. Mahue-Giangreco M, Mack W, Seligson H, Bourque LB. Risk factors associated with moderate and serious injuries attributable to the 1994 Northridge Earthquake, Los Angeles, California. Ann Epidemiol. 2001; 11(5):347-357.

45. Peek-Asa C, Ramirez M, Seligson H, Shoaf K. Seismic, structural, and individual factors associated with earthquake related injury. Inj Prev. 2003; 9(1):62-66.

46. Ramirez M, Peek-Asa C. Epidemiology of traumatic injuries from earthquakes. Epidemiol Rev. 2005;27:47-55.

47. Roces MC, White ME, Dayrit MM, Durkin ME. Risk factors for injuries due to the 1990 earthquake in Luzon, Philippines. Bull World Health Organ. 1992;70(4):509-514.

48. Seligson H, Shoaf K, Kano M. Development of casualty models for nonductile concrete frame structures for use in peer's performance-based earthquake engineering framework. Number 917. Paper presented at: 8th National Conference on Earthquake Engineering; 2006; San Francisco, CA.

49. Shoaf KI, Sareen HR, Nguyen LH, Bourque LB. Injuries as a result of California earthquakes in the past decade. Disasters. 1998;22(3):218-235.
50. Adedeji OA, Driscoll PA. The trauma team-a system of initial trauma care. Postgrad Med J. 1996;72(852):587-593.

51. Sugrue M, Seger M, Kerridge R, Sloane D, Deane S. A prospective study of the performance of the trauma team leader. J Trauma. 1995;38(1): 79-82.

52. Bergs EA, Rutten FL, Tadros T, Krijnen P, Schipper IB. Communication during trauma resuscitation: do we know what is happening? Injury. 2005; 36(8):905-911.

53. Cassera MA, Zheng B, Martinec DV, Dunst CM, Swanström LL. Surgical time independently affected by surgical team size. Am J Surg. 2009;198 (2):216-222.

54. Fung Kon Jin PH, van Olffen TB, Goslings JC, Luitse JS, Ponsen KJ. Inhospital downgrading of the trauma team: Validation of the Academic Medical Center downgrading criteria. Injury. 2006;37(1):33-40.

55. Hoff WS, Reilly PM, Rotondo MF, DiGiacomo JC, Schwab CW. The importance of the command-physician in trauma resuscitation. J Trauma. 1997;43(5):772-777.

56. Qazi K, Kempf JA, Christopher NC, Gerson LW. Paramedic judgment of the need for trauma team activation for pediatric patients. Acad Emerg Med. 1998;5(10):1002-1007.

57. Murrain-Hill P, Coleman CN, Hick JL, et al. Medical response to a nuclear detonation: creating a playbook for state and local planners and responders. Disaster Med Public Health Prep. 2011;5(Suppl 1):S89-S97.

58. van der Sluis CK, ten Duis HJ, Geertzen JH. Multiple injuries: an overview of the outcome. J Trauma. 1995;38(5):681-686.

59. Gabbe BJ, Cameron PA, Wolfe R, Simpson P, Smith KL, McNeil JJ. Predictors of mortality, length of stay and discharge destination in blunt trauma. ANZ J Surg. 2005;75(8):650-656.

60. Holtslag HR, Post MW, van der Werken C, Lindeman E. Return to work after major trauma. Clin Rehabil. 2007;21(4):373-383.

61. Stineman MG, Escarce JJ, Goin JE, Hamilton BB, Granger CV, Williams SV. A case-mix classification system for medical rehabilitation. Med Care. 1994;32(4):366-379.

62. Stineman MG, Escarce JJ, Tassoni CJ, Goin JE, Granger CV, Williams SV. Diagnostic coding and medical rehabilitation length of stay: their relationship. Arch Phys Med Rehabil. 1998;79(3):241-248.

63. Cameron CM, Purdie DM, Kliewer EV, McClure RJ. Ten-year health service use outcomes in a population-based cohort of 21,000 injured adults: the Manitoba injury outcome study. Bull World Health Organ. 2006;84 (10):802-810. 